\title{
A Combine-Correct-Combine Scheme for Optimizing Dissimilarity-Based Classifiers ${ }^{\star}$
}

\author{
Sang-Woon $\mathrm{Kim}^{1}$ and Robert P.W. Duin ${ }^{2}$ \\ 1 Dept. of Computer Science and Engineering, Myongji University, Yongin, \\ 449-728 South Korea \\ kimsw@mju.ac.kr \\ 2 Faculty of Electrical Engineering, Mathematics and Computer Science, \\ Delft University of Technology, The Netherlands \\ r.p.w.duin@tudelft.nl
}

\begin{abstract}
Recently, to increase the classification accuracy of dissimilarity-based classifications (DBCs), Kim and Duin [5] proposed a method of simultaneously employing fusion strategies in representing features (representation step) as well as in designing classifiers (generalization step). In this multiple fusion strategies, however, the resulting dissimilarity matrix is sometimes an indefinite one, causing problems in using the traditional pattern recognition tools after embedding the matrix in a vector space. To overcome this problem, we study a new way, named combine-correct-combine (CCC) scheme, of additionally employing an Euclidean correction procedure between the two steps. In CCC scheme, we first combine dissimilarity matrices obtained with different measures to a new dissimilarity representation using a representation combining strategy. Next, we correct the dissimilarity matrix using a pseudo-Euclidean embedding algorithm to improve the internal consistency of the matrix. After that, we again utilize the classifier combining strategies in the refined dissimilarity matrix to achieve an improved classification for a given data set. Our experimental results for well-known benchmark databases demonstrate that the CCC mechanism works well and achieves further improved results in terms of the classification accuracy compared with the previous multiple fusion approaches. The results especially demonstrate that the highest accuracies are obtained when the refined representation is classified with the trained combiners.
\end{abstract}

\section{Introduction}

In statistical pattern recognition, classification is performed in two steps: representation and generalization. In a case of dissimilarity-based classification (DBC) [9] 1], dissimilarity matrix is generated first from the training set in the representation step. Then, in the generalization step, classifiers are designed in the dissimilarity matrix.

\footnotetext{
* The work of the first author was done while visiting at Delft University of Technology, The Netherlands. We acknowledge financial support from the FET programme within the EU FP7, under the SIMBAD project (contract 213250). This work was supported by the Korea Research Foundation Grant funded by the Korean Government (KRF-2008-013-D00115).

${ }^{1}$ This methodology is not based on the feature measurements of the individual patterns, but rather on a suitable dissimilarity measure between them. An introduction to DBC will appear in a subsequent section.
}

E. Bayro-Corrochano and J.-O. Eklundh (Eds.): CIARP 2009, LNCS 5856, pp. 425-432. 2009.

(C) Springer-Verlag Berlin Heidelberg 2009 
On the other hand, combination systems which fuse "pieces" of information have received considerable attention because of its potential to improve the performance of individual systems [6], [7]. Recently, to increase the classification accuracy of DBCs, Kim and Duin [5] proposed a method of simultaneously employing multi-level fusion strategies in representing features (representation step) as well as in designing classifiers (generalization step). In [5], the authors first combined dissimilarity matrices obtained with different measures to a new representation matrix. Then, after training some base classifiers in the new representation matrix, they again combined the results of the base classifiers. In this multiple combining scheme, however, the representation matrix obtained is sometimes an indefinite one [8], causing problems in using the traditional pattern recognition tools after embedding the matrix in a vector space [10].

Combining dissimilarity matrices of metric measures sometimes leads to nonEuclidean ones, in which the metric requirement, such as the symmetry or the triangle inequality, is disobeyed. Duin and his colleagues [10] found that classifiers based on non-Euclidean dissimilarity representations may lead to better results than those based on transformed dissimilarity measures that are either Euclidean or have reduced non-Euclidean components [10]. Non-Euclidean vector spaces, however, are not well equipped with the tools for training classifiers; distances have to be computed in a specific way and are usually not invariant to orthogonal rotations. Also densities may not be properly defined, though some density-based classifiers can be used under some restrictions. So, Euclidean corrections called Euclideanization become of interest [8], [10].

To overcome the problem mentioned above, we study a new way, named combinecorrect-combine (CCC) scheme, of additionally employing an Euclidean correction procedure between the two steps. In CCC scheme, we first combine dissimilarity matrices obtained with different measures to a new representation matrix using one of the representation combining strategies. We then correct the representation matrix using pseudo-Euclidean embedding algorithms to improve the internal consistency of the matrix. Finally, we again utilize the classifier combining strategies in the refined dissimilarity matrix to achieve an improved classification accuracy. Indeed, we show that by correcting the dissimilarity representation matrix resulted from combining various dissimilarity matrices, we can obtain a refined representation matrix, using which, in turn, the classifier combining strategies can be employed again to improve the classification accuracy. Our experimental results for benchmark databases demonstrate that the proposed CCC mechanism works well and achieves further improved accuracies compared with the previous multiple fusion approaches [5].

The main contribution of this paper is to demonstrate that combined DBCs can be optimized by employing an Euclidean correction procedure. This has been done by executing the correction procedure prior to the classifier combining process and by demonstrating its strength in terms of the classification accuracy. The reader should observe that this philosophy is quite simple and distinct from that used in [5].

\section{Combine-Correct-Combine Scheme for DBCs}

Foundations of DBCs: A dissimilarity representation of a set of samples, $T=\left\{x_{i}\right\}_{i=1}^{n}$ $\in \Re^{d}$, is based on pairwise comparisons and is expressed, for example, as an $n \times m$ 
dissimilarity matrix $D_{T, Y}[i, j]$, where $Y=\left\{y_{j}\right\}_{j=1}^{m}$, a prototype set, is extracted from $T$, and the subscripts of $D$ represent the set of elements on which the dissimilarities are evaluated. Thus, each entry $D_{T, Y}[i, j]$ corresponds to the dissimilarity between the pairs of objects $\left\langle x_{i}, y_{j}\right\rangle$, where $x_{i} \in T$ and $y_{j} \in Y$. Consequently, an object $x_{i}$ is represented as a column vector as follows: $\left[d\left(x_{i}, y_{1}\right), d\left(x_{i}, y_{2}\right), \cdots, d\left(x_{i}, y_{m}\right)\right]^{T}, 1 \leq i \leq n$. Here, the measure $d$ is only required to be reflexive, i.e., $d(x, x)=0$ for all $x$. Also, the dissimilarity matrix $D$ is defined as a dissimilarity space on which the $d$-dimensional object, $x$, given in the feature space, is represented as an $m$-dimensional vector $\delta(x, Y)$, where if $x=x_{i}, \delta\left(x_{i}, Y\right)$ is the $i$-th row of $D$. In this paper, the dissimilarity matrix and its column vectors are simply denoted by $D(T, Y)$ and $\delta\left(x_{i}\right)$, respectively.

Representation Combining Strategies: Here, it is interesting to note that a number of distinct dissimilarity representations can be combined into a new one to obtain a more powerful representation in the discrimination. The idea of this feature combination is derived from the possibility that discriminative properties of different representations can be enhanced by a proper fusion [9] 2. There are several schemes for combining multiple representations to solve a given classification problem. Some of them are Average, Product, Min, and Max rules. For example, in the Average rule, two dissimilarity matrices, $D^{(1)}(T, Y)$ and $D^{(2)}(T, Y)$, can be averaged to $\left(\alpha_{1} D^{(1)}(T, Y)+\alpha_{2} D^{(2)}(T, Y)\right)$ after scaling with an appropriate weight, $\alpha_{i}$, to guarantee that they all take values in a similar range. The details of these methods are omitted here, but can be found in [9].

Representation Correcting Strategies: A symmetric dissimilarity matrix $D \in \Re^{n \times n}$ can be embedded in a pseudo-Euclidean space $\Xi\left(=\Re^{(p, q)}=\Re^{(p)} \oplus \Re^{(q)}\right)$, by an isometric mapping [3], [8], [9]. The pseudo-Euclidean space $\Xi$ is determined by eigendecomposition of an Gram matrix, $G=-\frac{1}{2} J D^{* 2} J$, derived from $D$, where $J$ is the centering matrix 3 and $D^{* 2}$ is the square dissimilarity matrix. The details of the derivation can be found in the related literature including [1], [9]. In this decomposition, $p$ positive and $q$ negative eigenvalues arise, indicating the signature of $\Xi$. The axes of $\Xi$ are constituted by $\sqrt{\left|\lambda_{i}\right|} \mu_{i}$, where $\lambda_{i}$ and $\mu_{i}$ are the $i$ th eigenvalue and the corresponding eigenvector of $G$, respectively.

There are several schemes for determining the pseudo-Euclidean space to refine the dissimilarity representation resulted from combining dissimilarity matrices. Some of them are briefly introduced as follows:

1. NON (non-refined space): This method is the same as the multiple fusion scheme in [5]. That is, the combiners are trained in non-refined matrix.

2. PES+ (pseudo Euclidean space): The most obvious correction for a pseudoEuclidean space $\Xi=\Re^{(p, q)}$ is to neglect the negative definite subspace. This discarding results in a $p$-dimensional Euclidean space $\Re^{(p)}$ with many-to-one mappings to $\Xi$. Consequently, it is possible that the class overlap increases.

3. AES (associated Euclidean space): Since $\Re^{(p, q)}$ is a vector space, we can keep all dimensions when performing the isometric mapping, which implies that the vector coordinates are identical to those of $\Xi$, but we now use the norm and distance measure that are Euclidean.

\footnotetext{
${ }^{2}$ This is also related to a kind of clustering ensemble which combines similarity matrices [13].

${ }^{3} J=I-\frac{1}{n} \mathbf{1 1}^{T} \in \Re^{n \times n}$, where $I$ is the identity matrix and $\mathbf{1}$ is an $n$-elements vector of all ones. The details of the centering matrix can be found in [1].
} 
4. AESR (associated Euclidean space reduction): In this correction, we can find an Euclidean subspace based on the $p^{\prime}(\leq p)$ positive eigenvalues and $q^{\prime}(\leq q)$ negative eigenvalues when computing a projection.

The details of the other correction procedures, such as DEC (dissimilarity enlargement by a constant), Relax (relaxation by a power transformation), and Laplace (Laplace transformation), are omitted here, but can be found in [3].

Classifier Combining Strategies: The basic strategy used in fusion is to solve the classification problem by designing a set of classifiers, and then combining the individual results obtained from these classifiers in some way to achieve reduced classification error rates. Therefore, the choice of an appropriate fusion method can further improve on the performance of the individual method. Various classifier fusion strategies have been proposed in the literature. Some of them are Product, Sum, Average, Max, Min, Median, Majority vote, and so on [6]. In addition, there are two commonly used approaches to implement multiple base-level classifiers; a fixed combiner and a trainable combiner. The fixed combiner has no extra parameter that need to be trained, while the trainable combiner needs additional training. For example, if a single training set is available, it is recommended to leave the base classifiers undertrained and subsequently complete the training of the combiner on the training set [7], [11]. Various classifier fusion strategies have been proposed in the literature - an excellent study is found in [7].

\section{Combined Dissimilarity-Based Classifiers (CDBCs)}

The reasons for combining several distinct dissimilarity representations and different dissimilarity-based classifiers will be investigated in the present paper. The proposed approach, which is referred to as a combined dissimilarity-based classifier (CDBC), is summarized in the following:

1. Select the input training data set $T$ as a representative subset $Y$.

2. Compute dissimilarity matrices, $D^{(1)}(T, Y), D^{(2)}(T, Y), \cdots, D^{(k)}(T, Y)$, by using the $k$ different dissimilarity measures for all $x \in T$ and $y \in Y$.

3. Combine the dissimilarity matrices, $\left\{D^{(i)}(T, Y)\right\}_{i=1}^{k}$, into new ones, $\left\{D^{(j)}\right.$ $(T, Y)\}_{j=1}^{l}$, by building an extended matrix or by computing their weighted average. Following this, correct the new matrices using an Euclidean correction procedure.

4. For any $D^{(j)}(T, Y),(j=1, \cdots, l)$, perform classification of the input, $\boldsymbol{z}$, with combined classifiers designed on the newly refined dissimilarity space as follows:

(a) Compute a dissimilarity column vector, $\delta^{(j)}(\boldsymbol{z})$, for the input sample $\boldsymbol{z}$, with the same method as in measuring the $D^{(j)}(T, Y)$.

(b) Classify $\delta^{(j)}(\boldsymbol{z})$ by invoking a group of DBCs as the base classifiers designed with $n m$-dimensional vectors in the dissimilarity space. The classification results are labeled as class $_{1}$, class $_{2}, \cdots$, respectively.

5. Obtain the final result from the class $_{1}$, class $_{2}, \cdots$, by combining the base classifiers designed in the above step, where the base classifiers are combined to form the final decision in the fixed or trained fashion.

The computational complexity of the proposed algorithm depends on the computational costs associated with the dissimilarity matrix. Thus, the time complexity and the space complexity of CDBC are $O\left(n^{2}+d^{3}\right)$ and $O(n(n+d))$, respectively. 


\section{Experimental Results}

Experimental Data: The proposed method has been tested by performing experiments on three benchmark databases, namely, the Yale 4, AT\&T 5 , and Nist38 databases.

The face database of Yale contains 165 gray scale images of 15 individuals. The size of each image is $243 \times 320$ pixels, for a total dimensionality of 77760 pixels. To reduce the computational complexity of this experiment, facial images of Yale database were down-sampled into $178 \times 236$ pixels and then represented by a centered vector of normalized intensity values. The face database of AT\&T consists of ten different images of 40 distinct subjects, for a total of 400 images. The size of each image is $112 \times 92$ pixels, for a total dimensionality of 10304 pixels. The data set captioned Nist38 chosen from the NIST database [12] consists of two kinds of digits, 3 and 8, for a total of 1000 binary images. The size of each image is $32 \times 32$ pixels, for a total dimensionality of 1024 pixels.

Experimental Method: All our experiments were performed with a leave-one-out (LOO) strategy. To classify an image of an object, we removed the image from the training set and computed the dissimilarity matrix with the $n-1$ images. This process was repeated $n$ times for every image, and a final result was obtained by averaging the results of each image. To compute the dissimilarity matrix, we first selected all training samples as the representative. We then measured the dissimilarities between them using four systems: Euclidean distance (ED), Hamming distance (HD) 6 , the regional distance (RD) 7 , and the spatially weighted gray-level Hausdorff distance (WD) 8 measures.

First of all, to investigate the representation combination, we experimented with three Average methods: Ex-1, Ex-2, and Ex-3. In Ex-1, two dissimilarity matrices obtained with ED and RD measures are averaged to a new representation after normalization, where the scaling factors are $\alpha_{i}=\frac{1}{2}, i=1,2$. In Ex-2, three dissimilarity matrices obtained with ED, RD, and HD measures are averaged to a new one with $\alpha_{i}=\frac{1}{3}, i=$ 1, 2,3. In Ex-3, four dissimilarity matrices measured with ED, RD, HD, and WD are averaged with $\alpha_{i}=\frac{1}{4}, i=1, \cdots, 4$. In general, $\alpha_{i}=\frac{1}{N}$, where $N$ is the number of matrices to be combined.

Next, to improve the internal consistency of the combined matrices, we refined them with three kinds of Euclidean corrections: AES, PES+, and AESR. In AES, all dimensions are kept when mapping the matrix onto a pseudo-Euclidean subspace. In PES+,

\footnotetext{
${ }^{4}$ http://www1.cs.columbia.edu/ belhumeur/pub/images/yalefaces

${ }^{5} \mathrm{http} / / /$ www.cl.cam.ac.uk/Research/DTG/attarchive/facedatabase.html

${ }^{6}$ Hamming distance between two strings of equal length is the number of positions for which the corresponding symbols are different. For binary strings $\alpha$ and $\beta$, for example, the Hamming distance is equal to the number of ones in $\alpha \oplus \beta$ ( $\oplus$ means Exclusive-OR operation).

${ }^{7}$ The regional distance is defined as the average of the minimum difference between the gray value of a pixel and the gray value of each pixel in a $5 \times 5$ neighborhood of the corresponding pixel. In this case, the regional distance compensates for a displacement of up to three pixels of the images.

${ }^{8}$ In WD, we compute the dissimilarity directly from input gray-level images without extracting the binary edge images from them. Also, instead of obtaining the distance on the basis of the entire image, we use a spatially weighted mask, which divides the image region into several subregions according to their importance.
} 

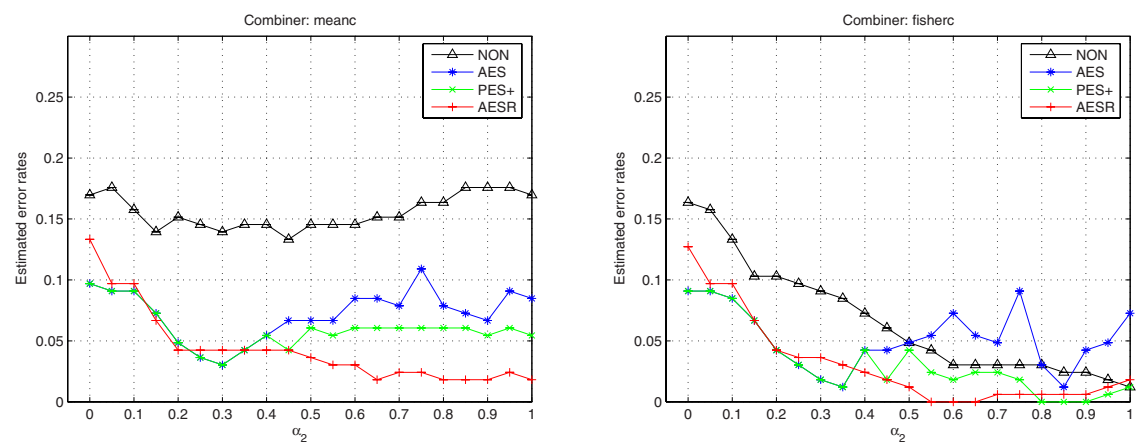

Fig. 1. A comparison of the error rates of two combiners, meanc and fisherc, obtained with Ex-1 method for Yale. Here $\alpha_{2}\left(=1-\alpha_{1}\right)$ is the scaling factor of the dissimilarity matrix. Four markers describing the same Euclidean corrections are connected by lines to enhance the visibility.

a subspace is found based on the positive eigenvalues only, while, in AESR, the subspace is found such that at least a fraction of the negative eigenvalues is preserved. For instance, in this experiment, the cumulative fractions of the positive and negative eigenvalues were 0.9 and 0.1 , respectively.

Finally, in CDBCs, $l$ was set as 1 ; only one combination of dissimilarity matrices was made. Also, after training three base classifiers, all of the results were combined in trainable fashion. Here, the three base classifiers and the three trained combiners were implemented with PRTools 9 , and named $n m c, l d c$, knnc, meanc, fisherc, and naivebc, respectively.

Experimental Results: First of all, to examine the rationality of employing the refining techniques in CDBCs, the classification error rates of two combiners, meanc and fisherc, were evaluated with Ex-1 method for Yale database. Here, combining dissimilarity matrices was done with 21 different scaling factors; $\alpha_{2}=0.0,0.05, \cdots, 1.0$ and $\alpha_{1}=1-\alpha_{2}$. Then, the resulted matrices were corrected in three ways: AES, PES+, and AESR. Finally, the results of the base classifiers were combined in trained fashion. Fig. 1 shows a comparison of the error rates obtained with Ex-1 method for Yale.

From the figure, it should be observed that the classification accuracies of the combiners trained in the refined matrix can be improved. This is clearly shown from the results of meanc (see the left picture), where the error rates of a NON-refined matrix is shown with the connected lines of $\triangle$ marker, and those of three refined matrices with $*, \times$, and + markers. Similar, not necessarily the same, characteristics could also be observed in the results of fisherc (see the right picture). The details of the results are omitted here in the interest of compactness.

In order to further investigate the advantage gained with utilizing the $\mathrm{CCC}$ scheme, we repeated the experiments (of estimating error rates) in Ex-1, Ex-2, and Ex-3. Table 1 shows the estimated error rates of CDBCs designed as the base classifiers and the trainable combiners for the three experimental databases. In the table, the values underlined are the lowest ones in the 24 error rates (12 for the base classifiers and 12 for the trained combiners per each fusion method).

${ }^{9}$ PRTools is a Matlab toolbox for pattern recognition (refer to http://prtools.org/). 
Table 1. A comparison of the estimated error rates of DBCs designed as the base classifiers and the trainable combiners for the three databases, where the values underlined are the lowest ones in the 24 error rates of the base and trained combiners per each combining method

\begin{tabular}{|c|c|c|c|c|c|c|c|c|}
\hline \multirow{2}{*}{$\begin{array}{c}\text { experimental } \\
\text { databases }\end{array}$} & \multirow{2}{*}{$\begin{array}{l}\text { combining } \\
\text { method }\end{array}$} & \multirow{2}{*}{$\begin{array}{c}\text { correcting } \\
\text { method }\end{array}$} & \multicolumn{3}{|c|}{ base classifiers } & \multicolumn{3}{|c|}{ combiners } \\
\hline & & & $n m c$ & ldc & knnc & meanc & fisherc & naivebc \\
\hline \multirow{12}{*}{ Yale } & \multirow{4}{*}{ Ex-1 } & NON & 0.2242 & 0.0485 & 0.1939 & 0.1455 & 0.0485 & 0.1455 \\
\hline & & AES & 0.1030 & 0.0485 & 0.1879 & 0.0667 & 0.0485 & 0.1091 \\
\hline & & PES+ & 0.1091 & 0.0424 & 0.1879 & 0.0606 & 0.0424 & 0.1030 \\
\hline & & AESR & 0.1091 & 0.0121 & 0.1939 & 0.0364 & 0.0121 & 0.0970 \\
\hline & \multirow{4}{*}{ Ex-2 } & $\mathrm{NON}$ & 0.2061 & 0.0909 & 0.1939 & 0.1455 & 0.0909 & 0.1697 \\
\hline & & AES & 0.1273 & 0.0182 & 0.1879 & 0.0424 & 0.0182 & 0.1091 \\
\hline & & PES+ & 0.1273 & 0.0182 & 0.1879 & 0.0424 & 0.0182 & 0.1091 \\
\hline & & AESR & 0.1273 & $\overline{0.0303}$ & 0.1939 & 0.0424 & $\overline{0.0303}$ & 0.1152 \\
\hline & \multirow{4}{*}{ Ex-3 } & $\overline{\mathrm{NON}}$ & 0.2121 & 0.0485 & 0.2121 & 0.1576 & 0.0485 & 0.1818 \\
\hline & & AES & 0.1576 & 0.0848 & 0.2061 & 0.1273 & 0.0848 & 0.1697 \\
\hline & & PES+ & 0.1576 & 0.0485 & 0.1879 & 0.0970 & 0.0485 & 0.1576 \\
\hline & & AESR & 0.1576 & 0.0121 & 0.2061 & 0.0485 & 0.0121 & 0.1515 \\
\hline \multirow{12}{*}{ AT\&T } & \multirow{4}{*}{ Ex-1 } & NON & 0.2350 & 0.0075 & 0.0425 & 0.0300 & 0.0075 & 0.0300 \\
\hline & & AES & 0.0600 & 0.0250 & 0.0175 & 0.0275 & 0.0325 & 0.0300 \\
\hline & & PES+ & 0.0575 & 0.0525 & 0.0150 & 0.0300 & 0.0500 & 0.0250 \\
\hline & & AESR & 0.0700 & 0.0150 & 0.0175 & 0.0175 & 0.0250 & 0.0225 \\
\hline & \multirow{4}{*}{ Ex-2 } & $\mathrm{NON}$ & 0.1950 & 0.0050 & 0.0225 & 0.0175 & 0.0050 & 0.0175 \\
\hline & & AES & 0.0375 & 0.0350 & 0.0050 & 0.0125 & 0.0300 & 0.0100 \\
\hline & & PES+ & 0.0375 & 0.0550 & 0.0050 & 0.0150 & 0.0475 & 0.0125 \\
\hline & & AESR & 0.0375 & $\underline{0.0050}$ & 0.0075 & $\underline{0.0050}$ & $\underline{0.0050}$ & 0.0075 \\
\hline & \multirow{4}{*}{ Ex-3 } & $\mathrm{NON}$ & 0.2125 & 0.0050 & 0.0250 & 0.0175 & 0.0075 & 0.0175 \\
\hline & & AES & 0.0375 & 0.0675 & 0.0050 & 0.0150 & 0.0475 & 0.0125 \\
\hline & & PES+ & 0.0375 & 0.0925 & 0.0075 & 0.0150 & 0.0700 & 0.0150 \\
\hline & & AESR & 0.0450 & 0.0025 & 0.0100 & 0.0025 & 0.0025 & 0.0100 \\
\hline \multirow{12}{*}{ Nist38 } & \multirow{4}{*}{ Ex-1 } & $\overline{\mathrm{NON}}$ & 0.1220 & 0.0220 & 0.0190 & 0.0190 & 0.0190 & 0.0220 \\
\hline & & AES & 0.0890 & 0.0670 & 0.0110 & 0.0370 & 0.0660 & 0.0220 \\
\hline & & PES+ & 0.0930 & 0.0610 & 0.0130 & 0.0460 & 0.0130 & 0.0190 \\
\hline & & AESR & 0.0930 & 0.0260 & $\underline{0.0110}$ & 0.0250 & 0.0110 & 0.0110 \\
\hline & \multirow{4}{*}{ Ex-2 } & $\mathrm{NON}$ & 0.1230 & 0.0210 & 0.0170 & 0.0170 & 0.0170 & 0.0220 \\
\hline & & AES & 0.0900 & 0.1060 & 0.0110 & 0.0490 & 0.1060 & 0.0210 \\
\hline & & PES+ & 0.0920 & 0.0720 & 0.0150 & 0.0490 & 0.0150 & 0.0160 \\
\hline & & AESR & 0.0910 & 0.0290 & $\underline{0.0120}$ & 0.0240 & $\underline{0.0120}$ & 0.0120 \\
\hline & \multirow{4}{*}{ Ex-3 } & $\mathrm{NON}$ & 0.1660 & 0.0180 & 0.0270 & 0.0180 & 0.0270 & 0.0270 \\
\hline & & AES & 0.0990 & 0.1990 & 0.0140 & 0.1010 & 0.1990 & 0.0350 \\
\hline & & PES+ & 0.1010 & 0.1120 & 0.0140 & 0.0560 & 0.0140 & 0.0170 \\
\hline & & AESR & 0.0990 & 0.0240 & 0.0140 & 0.0180 & 0.0140 & 0.0130 \\
\hline
\end{tabular}

The observations obtained from the table are the followings:

- The best Ex-3 results are usually better than the best Ex-2 and Ex-1 results, so combining dissimilarity matrices is helpful.

- The corrected results (AES, PES+ and AESR) are sometimes better than the original results (NON). So correction can be helpful, but sometimes it is not.

- The results fisherc as a trainable combining classifier are about equal to those of the best base classifier (usually ldc, sometimes knnc) and it thereby operates as a selector. Consequently, the combining classifier makes the system more robust.

Finally, we didn't present standard deviations in Table 1 to save some space as we don't claim that some improvements are significant. A more robust analysis can be performed in terms of quantitative measures such as the kappa or tau coefficients [2]. 


\section{Conclusions}

In this paper, we proposed to utilize the combine-correct-combine (CCC) scheme to optimize dissimilarity-based classifications (DBCs). The CCC scheme involves a step wherein the combined dissimilarity matrix is corrected prior to employing the classifier combining strategies to improve the internal consistency of the dissimilarity matrix. The presented experimental results for three benchmark databases demonstrate that the studied CCC mechanism works well and achieves robust, good results. Despite this success, problems remain to be addressed. First, classification performance could be improved furthermore by developing an optimal Euclidean correction and by designing suitable combiners in the refined dissimilarity space. Then, the experimental results also show that the highest accuracies are achieved when the refined representation is classified with the trained combiners. The problem of theoretically analyzing this observation remains unresolved. Future research will address these concerns.

\section{References}

1. Borg, I., Groenen, P.: Morden Mutlidimensional Scaling: Theory and Applications. Springer, New York (1997)

2. Congalton, R.G.: A review of assessing the accuracy of classifications of remotely sensed data. Remote Sensing of Enviroment 37, 35-46 (1991)

3. Duin, R.P.W., Pekalska, E., Harol, A., Lee, W.: On Euclidean corrections for non-Euclidean dissimilarities. In: da Vitoria Lobo, N., Kasparis, T., Roli, F., Kwok, J.T., Georgiopoulos, M., Anagnostopoulos, G.C., Loog, M. (eds.) S+SSPR 2008. LNCS, vol. 5342, pp. 551-561. Springer, Heidelberg (2008)

4. Haasdonk, H., Burkhardt, B.: Invariant kernels for pattern analysis and machine learning. Machine Learning 68, 35-61 (2007)

5. Kim, S.-W., Duin, R.P.W.: On optimizing dissimilarity-based classifier using multi-level fusion strategies. Journal of The Institute of Electronics Engineers of Korea 45-CI(5), 15-24 (2008) (in korean); A preliminary version of this paper was presented at the 20th Canadian Conference on Artificial Intelligence, Montreal, Canada. LNCS (LNAI), vol. 4509, pp. 110-121 (2007)

6. Kittler, J., Hatef, M., Duin, R.P.W., Matas, J.: On combining classifiers. IEEE Trans. Pattern Anal. and Machine Intell. 20(3), 226-239 (1998)

7. Kuncheva, L.I.: Combining Pattern Classifiers - Methods and Algorithms. John Wiley \& Sons, New Jersey (2004)

8. Munoz, A., de Diego, I.M.: From indefinite to positive semi-definite matrices. In: Yeung, D.-Y., Kwok, J.T., Fred, A., Roli, F., de Ridder, D. (eds.) SSPR 2006 and SPR 2006. LNCS, vol. 4109, pp. 764-772. Springer, Heidelberg (2006)

9. Pekalska, E., Duin, R.P.W.: The Dissimilarity Representation for Pattern Recognition: Foundations and Applications. World Scientific Publishing, Singapore (2005)

10. Pekalska, E., Harol, A., Duin, R.P.W., Spillmann, B., Bunke, H.: Non-Euclidean or nonmetric measures can be informative. In: Yeung, D.-Y., Kwok, J.T., Fred, A., Roli, F., de Ridder, D. (eds.) SSPR 2006 and SPR 2006. LNCS, vol. 4109, pp. 871-880. Springer, Heidelberg (2006)

11. Todorovski, L., Dzeroski, S.: Combining classifiers with meta decision trees. Machine Learning 50(3), 223-249 (2003)

12. Wilson, C.L., Garris, M.D.: Handprinted Character Database 3, Technical report, National Institute of Standards and Technology, Gaithersburg, Maryland (1992)

13. Zhou, Z.-H., Tang, W.: Clusterer ensemble. Knowledge-Based System 19, 77-83 (2006) 